\title{
Singularity Analysis of 3 DOF Parallel Manipulators for Angular Drilling in Medical Applications
}

\author{
Arockia Selvakumar A
}

\begin{abstract}
This paper focuses on two 3 - PRS (Prismatic Revolute - Spherical) parallel manipulators (PMs) carried out by considering three type of singularities. For the study, pin joint forces and torques, linear displacement of nut and mobile platform (MP) tilt of TRIPOD and TRI-GLIDE PMs are taken as important parameters. The singularity positions of the parallel manipulators are simulated in ADAMS environment. The geometrical parameters of the parallel manipulators are considered in logical approach. Based on the results, the concluding remarks are summarized at the end to design the 3 PRS parallel manipulators with better singular positions for angular drilling in medical applications.
\end{abstract}

Keywords: ADAMS, Parallel Manipulator, Singularity, TRIPOD, TRI-GLIDE

\section{INTRODUCTION}

$\mathrm{I}_{\mathrm{n}}$ recent years, the robotic industry needs Parallel Manipulator for machining and manufacturing process due to higher precision, robustness, stiffness and load-carrying capacity $[11][12][13][15][16][17]$. Most of the PMs kinematics solutions are affected due to their structure or configuration in singular position [1]. PMs precision and control will be lost at singularity condition and PM will gain one or more DOF. Based on the geometrical parameters base, legs and planes, three types of singularity analysis were presented using forward and inverse Jacobian for the non identical 3 DOF PMs and the PMs designs are used in Parallel kinematic machines and Simulators [2]. The PM performance is improved by reshaping the singularities with the help of redundant actuation [5]. In singularity analysis, joints like Translation $(\mathrm{T})$ and Rotational $(\mathrm{R})$ are used to achieve PM motion range by proposing various joint assembly configurations [6]. Similarly, the singular configurations and conditions are found based on the displacements of joints [8]. Clustering algorithm and local routing methods are proposed to achieve PM path planning without singularity [4]. Screw theory is presented with actuation and limb singularities which can be used for the analysis of symmetrical PMs [7]. Grassman-Cayley Algebra is implemented to analyze the parallel singularities of less than 6 DOF PMs and the

Revised Manuscript Received on December 20, 2019.

* Correspondence Author

Dr. A. Arockia Selvakumar*, Senior Associate Professor, Design and Automation Research group, School of Mechanical and Building Sciences, Vellore Institute of Technology, Chennai, Tamil Nadu, India.

Email: arockiaselvakumar@yahoo.co.in singularities are characterized by geometrically [9][10]. Eleven different positions of singularities were shown based on three types of singularities [3]. The position analysis of PMs is carried out by analytical form and the mobile platform motions are found to be zero at direct singularities [11]. Singularity analysis of spherical, revolute PMs is modeled and simulated using a CAD package SMAPS and the results are found to be satisfactory [12].

The singularity analysis and simulation of parallel manipulators have been studied so far in the literature. The papers on the singularity analysis of parallel manipulators accounted for both mobility, work space, and the constraints of the parallel configurations were also considered. The studies of the singularity analysis in the simulation of parallel configurations are very few in the open literature. This work investigates the effect of the mobile platform radius, link length and initial angle between the link and the base platform on the singular positions of some specific parallel manipulator units. By varying one or more of the geometrical parameters of the system, the design of the parallel manipulators can be brought closer to achieve better singular positions. In this paper, the definition of the parallel manipulator and the architectural description of PMs are presented in the following sections. Furthermore, the singularity analyses based on three positions are also explained. This is followed by simulation results of two manipulators are discussed. Finally, the concluding remarks of the comparative study are summarized.

\section{Architectural Description of Parallel MANIPULATORS}

The 3-DOF Tripod and Tri-glide PMs are shown in Figures 1 and 2. The PMs are typically consisting of a Mobile Platform or Moving platform (MP), Base Platform (BP) and links. The mobile platform is attached to the base platform through joints and links. Revolute joints are used to connect the BP with the links; similarly spherical joints are used to connect the MP with links. The nuts are mounted on the guide ways which consists of two guide rods and a leadscrews. Further the nuts are attached with the revolute joints [12] [13][15][16]. The geometrical parameters of the two PMs are taken by logical approach for the angular drilling in medical applications.

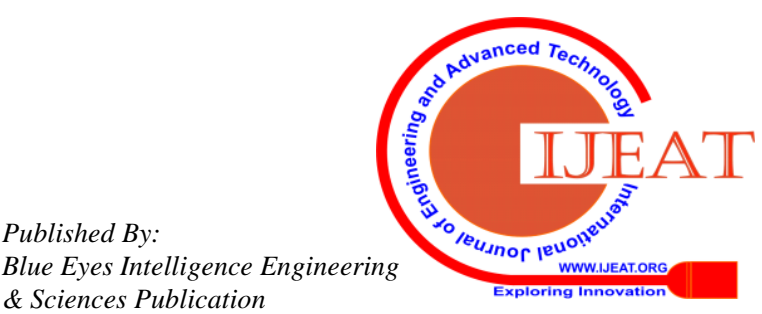




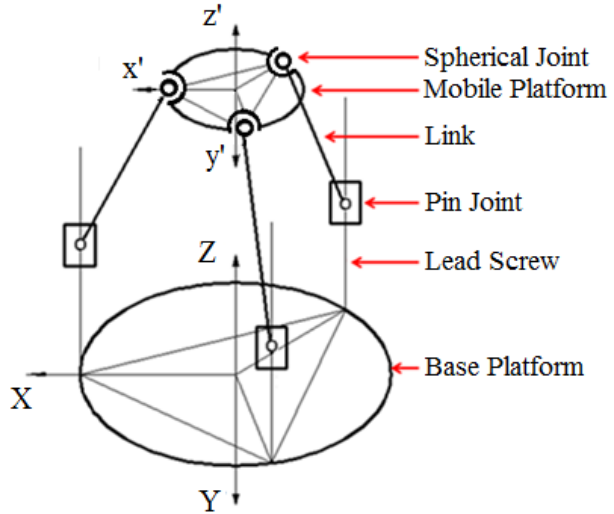

Fig. 1. Tripod PM

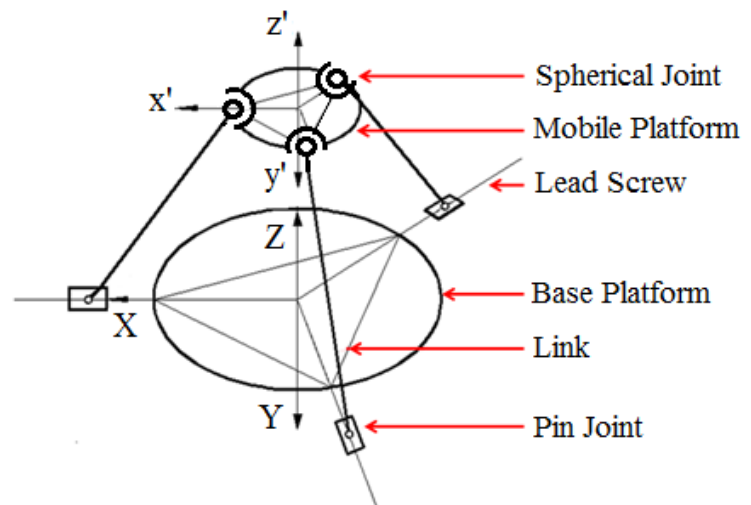

Fig. 2. Tri-Glide PM

\section{SINGULARITY ANALYSIS}

Singularity is a significant problem in parallel mechanisms, because it leads to loss of controllability and degradation of the stiffness of the system [14][15]. The Figures 3 and 4 shows the schematic diagram of Type I and Type II Singularity positions of PMs [15].

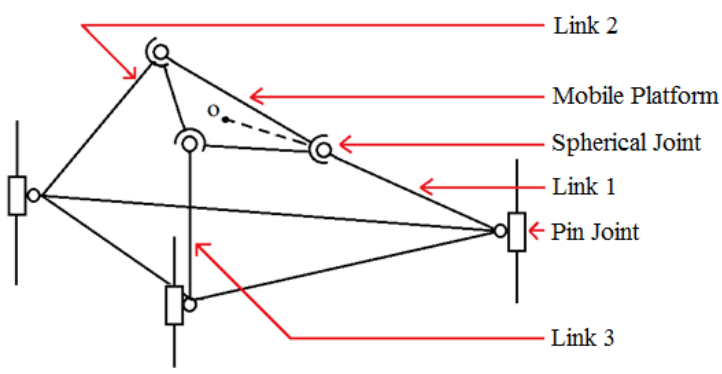

Fig. 3. Singular Poses - Type I Singularity

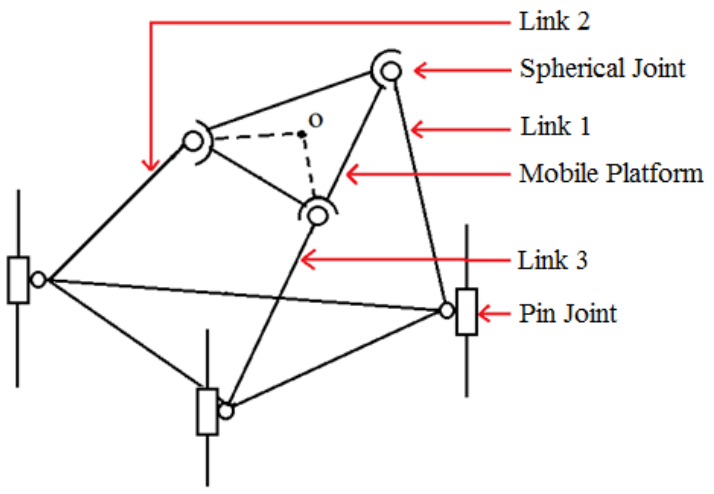

Fig. 4. Singular Poses - Type II Singularity
Type I Singularity position shows the posture of the link 1 and the mobile platform being in the same plane. Similarly, the posture of link 2 , link 3 and the mobile platform being in the same plane in the Type II Singularity position [6]. The proposed mechanisms are simulated to find the various singularities, and the positions of the PMs are as shown in Figures 5a to f. Figures 5a and 5d show the first kind (Type I) of singularity of the PRS PMs, when the MP has $180^{\circ}$ with any one of the links. Similarly, the MP is in line with the other two links (L2, L3) while link (L1) is kept constant [15]. The second kind (Type II) of singularity of the mechanism is obtained as shown in Figures $5 \mathrm{~b}$ and $5 \mathrm{e}$. The third kind (Type III) of singularity is obtained when all the links are actuated in different positions to reach the first or second type of singularity position as shown in Figure $5 \mathrm{c}$ and $5 \mathrm{f}$. The above mentioned three kinds of singularities are taken in to consideration for the comparative study of the two PMs.

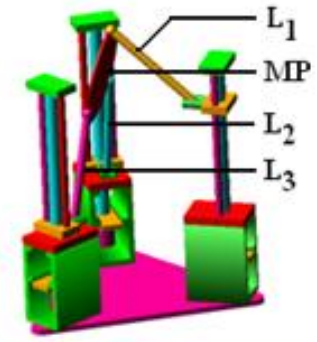

a

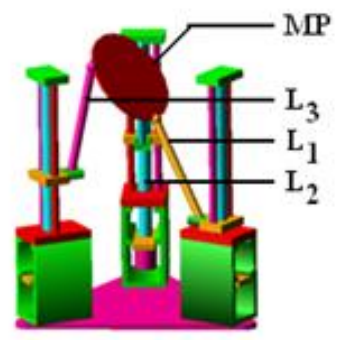

b
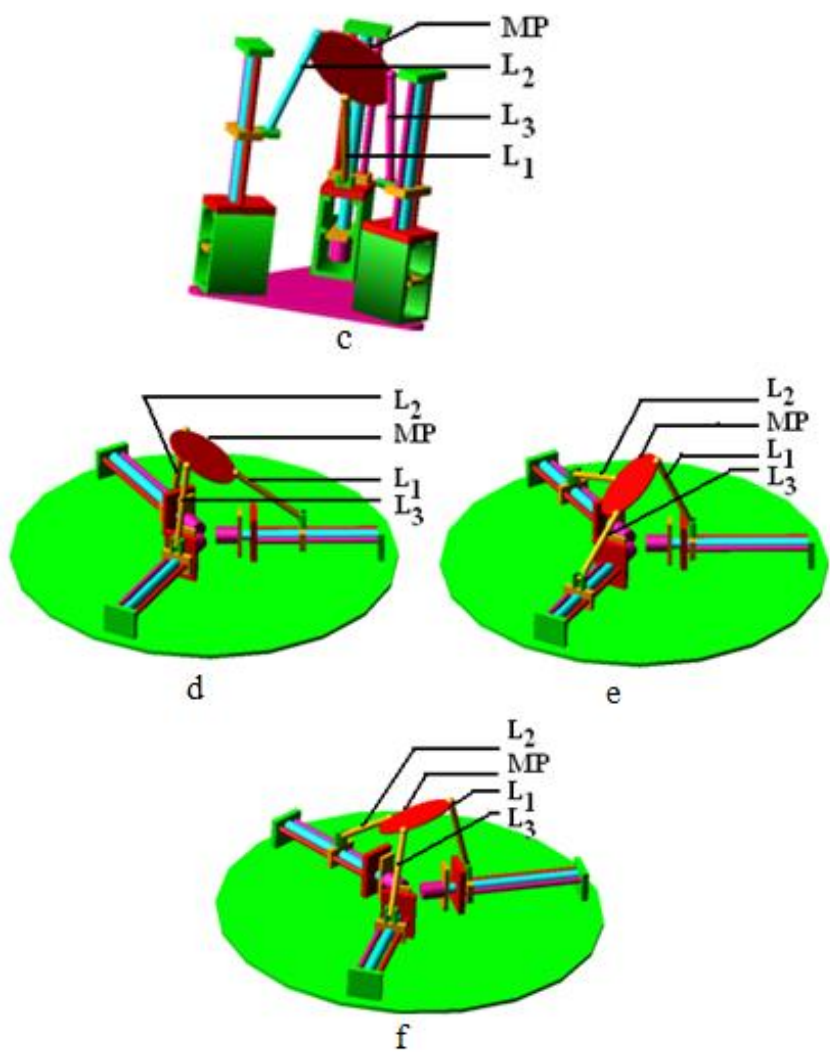

Fig. 5. Three kinds of singularity positions a) Tripod Type I singularity b) Tripod Type II singularity c) Tripod Type III singularity d) Tri-Glide Type I singularity e) Tri-Glide Type II singularity f) Tri-Glide Type III singularity 


\section{RESULTS AND DISCUSSION}

The Tripod and Tri-Glide PMs are considered for the singularity analysis by considering pin joint forces and torques, linear displacement of nut and Mobile platform (MP) tilt for angular drilling in medical applications. For this simulation study, the geometrical parameters of Tripod and Tri-Glide PMs are considered by logical approach. The geometrical parameters are, MP radius $=90 \mathrm{~mm}$, Link length $=200 \mathrm{~mm}$ and the initial angle between the link and the base platform $=77^{\circ}$

Table 1 shows the simulation results for the two 3-DOF PMs with their MP tilt for the singular positions. From the results, it can be observed that in Type I singularity, the Tripod and the Tri-Glide MP tilts are found to be $78.97^{\circ}$ and $34.58^{\circ}$ about the $\mathrm{X}$-axis $(\alpha)$. Similarly, in Type II singularity, the Tripod and the Tri-Glide MP tilts are found to be $53.41^{\circ}$ and $75.73^{\circ}$ about the $\mathrm{X}$-axis. In Type III singularity, the Tripod and the Tri-Glide MP tilts are found to be $8.24^{\circ}$ and $10.28^{\circ}$ about the $\mathrm{X}$-axis and $59.1^{\circ}$ and $30.74^{\circ}$ about the $\mathrm{Y}$-axis $(\beta)$.

Table- I: Results of the MP tilt and nut displacement at singular positions

\begin{tabular}{|c|c|c|c|c|c|c|}
\hline \multirow{2}{*}{ Parameters } & \multicolumn{3}{|c|}{ Tripod } & \multicolumn{3}{|c|}{ Tri-Glide } \\
\cline { 2 - 7 } & I & II & III & I & II & III \\
\hline$\alpha$ in $^{\circ}$ & 78.97 & 53.41 & 8.24 & 34.58 & 75.73 & 10.28 \\
\hline$\beta$ in $^{\circ}$ & 0 & 0 & 59.1 & 0 & 0 & 30.74 \\
\hline $\begin{array}{c}\text { D1 } \\
(\mathrm{mm})\end{array}$ & 200 & 0 & 60 & 94 & 0 & 50 \\
\hline $\begin{array}{c}\mathrm{D} 2 \\
(\mathrm{~mm})\end{array}$ & 0 & 88 & 120 & 0 & 156 & 100 \\
\hline $\begin{array}{c}\mathrm{D} 3 \\
(\mathrm{~mm})\end{array}$ & 0 & 88 & 0 & 0 & 156 & 0 \\
\hline
\end{tabular}

The linear displacement of the nut 1(D1) for the Type I singularity is found to be $200 \mathrm{~mm}$ and $94 \mathrm{~mm}$ respectively for the Tripod and Tri-Glide PMs. Similarly, the linear displacement of the nuts 2 and 3 for the Type II singularity is found to be $88 \mathrm{~mm}$ for the Tripod PM and $156 \mathrm{~mm}$ for the Tri-Glide PM. In Type III singularity, the linear displacement of the nuts 1 and 2 is found to be $60 \mathrm{~mm}$ and $120 \mathrm{~mm}$ for the Tripod PM and $50 \mathrm{~mm}$ and $100 \mathrm{~mm}$ for the Tri-Glide PM.

Figures 6 and 17 show the result of the pin joints (PJs) force and torque values of the two PMs. From the results, it can be observed that the Tri-Glide PM and the Tripod PM have the joint force of $117 \mathrm{~N}$ and $8.84 \mathrm{~N}$ for Type I singularity. Similarly, for Type II singularity the Tri-Glide and Tripod PMs have $288.99 \mathrm{~N}$ and $24 \mathrm{~N}$. For the Type III singularity Tri-Glide and Tripod PMs have $54.32 \mathrm{~N}$ and $35 \mathrm{~N}$.

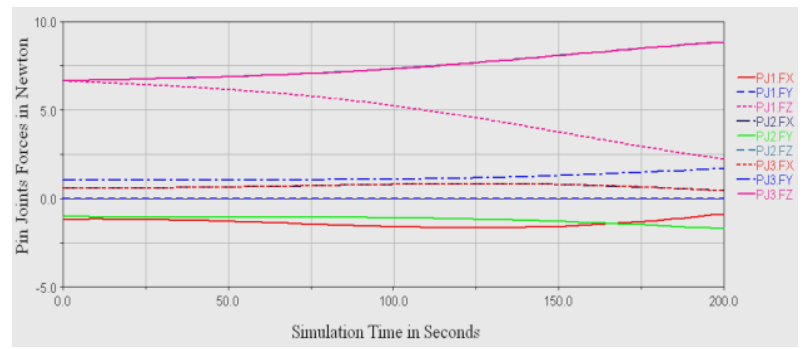

Fig. 6. Type I singularity pin joints force (Tripod)

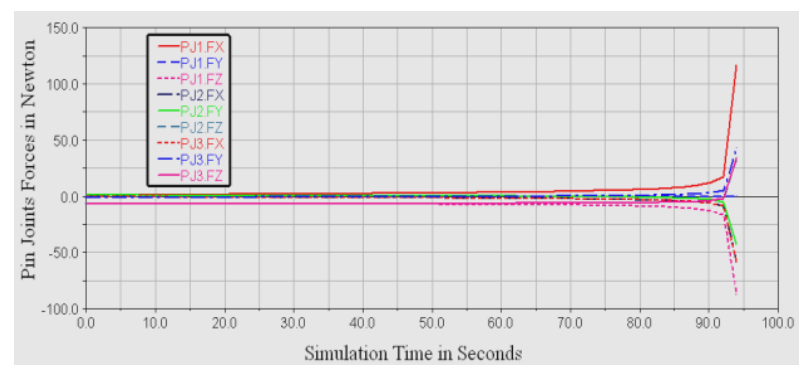

Fig. 7. Type I singularity pin joints force (Tri-Glide)

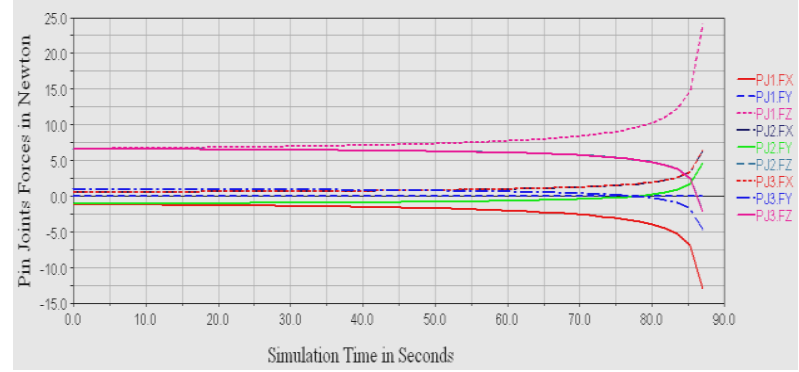

Fig. 8. Type II singularity pin joints force (Tripod)

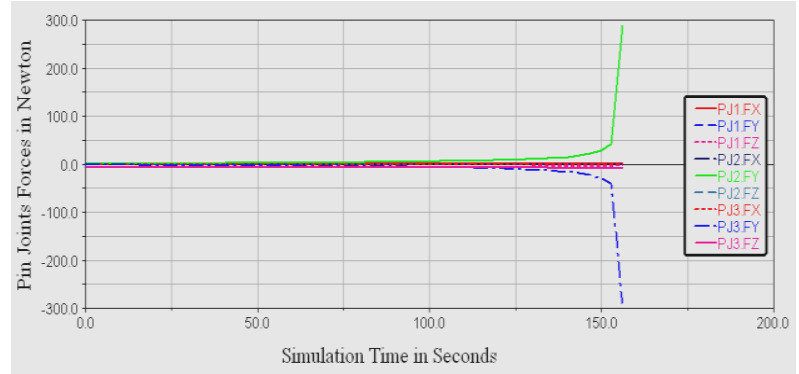

Fig. 9. Type II Singularity pin joints force (Tri-Glide)

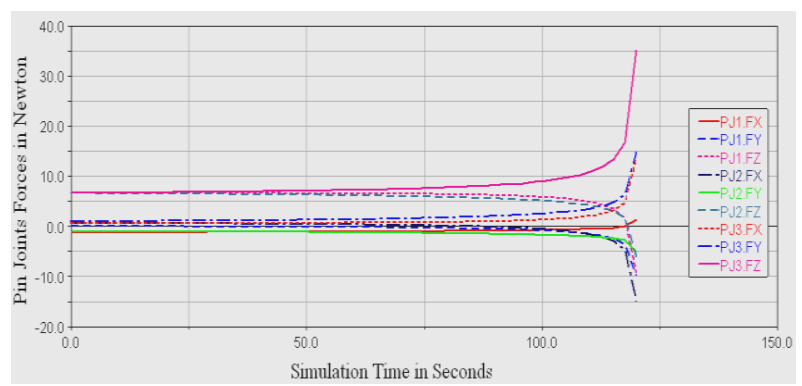

Fig. 10. Type III singularity pin joints force (Tripod)

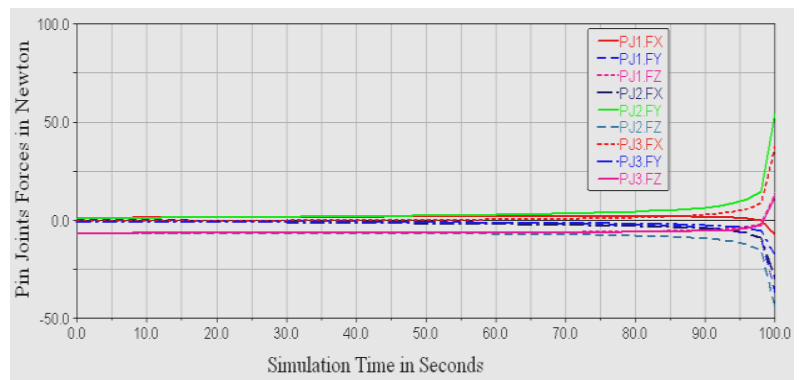

Fig. 11. Type III singularity pin joints force (Tri-Glide) 


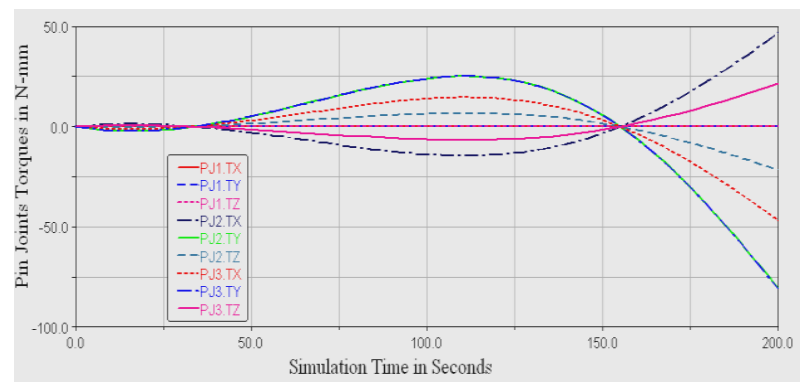

Fig. 12. Type I singularity pin joints Torque (Tripod)

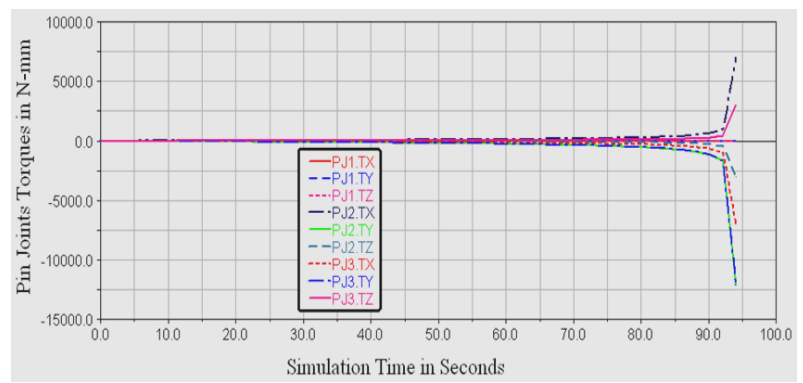

Fig. 13. Type I singularity pin joints torque (Tri-Glide)

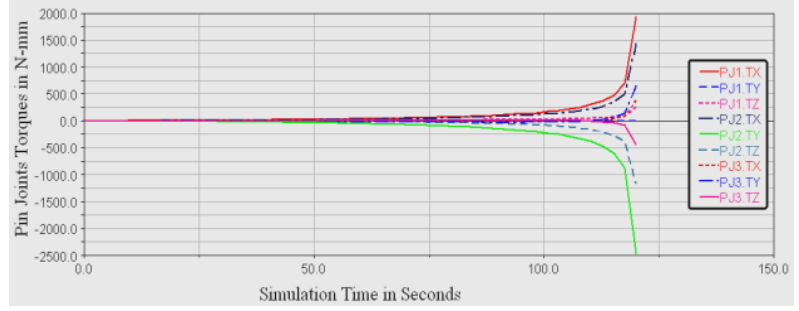

Fig. 14. Type II singularity pin joints torque (Tripod)

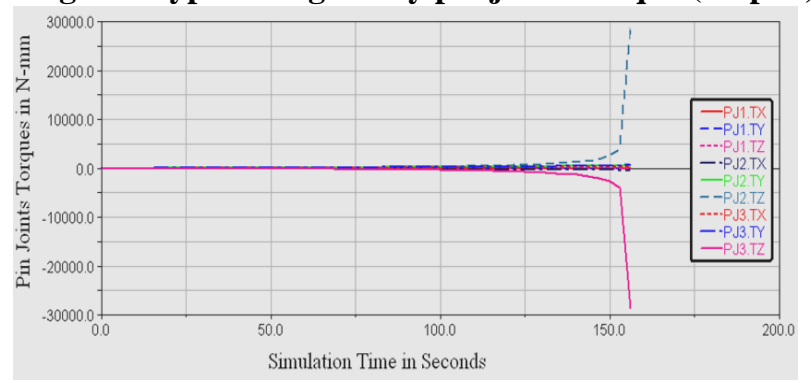

Fig. 15. Type II singularity pin joints torque (Tri-Glide)

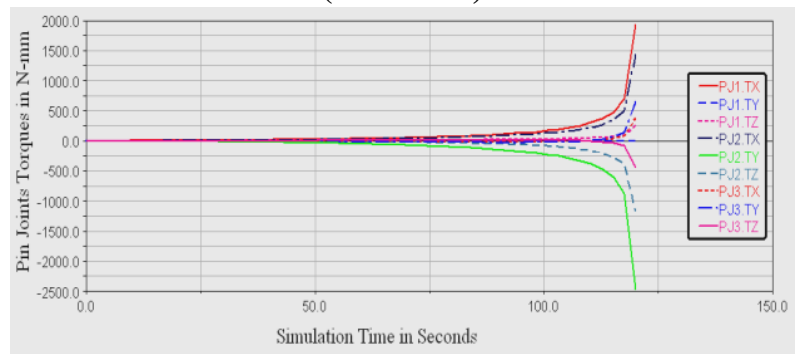

Fig. 16. Type III singularity pin joints torque (Tripod)

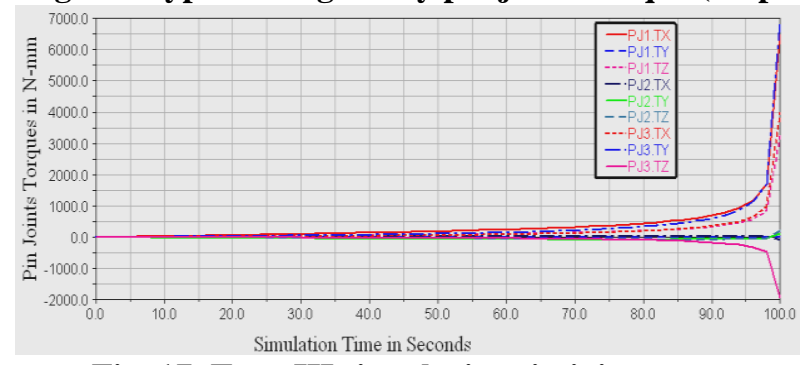

Fig. 17. Type III singularity pin joints torque

(Tri-Glide)
Similarly, the pin joints torque values of the Tri-Glide and Tripod PMs are found to be 12056.90 and $80.84 \mathrm{~N} \mathrm{~mm}$ for Type I singularity. For the Type II singularity, the values are found to be 28531.65 and $1336.29 \mathrm{~N} \mathrm{~mm}$. The pin joints torque values of the Tri-Glide and Tripod PMs, for Type III singularity are found to be 6879.09 and $2459.13 \mathrm{~N} \mathrm{~mm}$.

\section{CONCLUSION}

The investigations of this study provided an insight into the singularity analyses of 3-DOF PMs to design a manipulator with better singularity positions. The conclusions of the study based on the three types of singularity positions of TRIPOD and TRI-Glide PMs for angular drilling in medical applications are summarized below.

- From the singularity study, it is observed that the Tri-Glide PM has $53 \%$ lesser linear displacement of the nut than the Tripod PM for the Type I singularity with a single link movement. Similarly, the Tripod PM has $43.5 \%$ lesser linear displacement of the nut than the Tri-Glide PM for the Type II singularity with two links (simultaneous) movement.

- The Tripod PM MP tilt is found to be $56.27 \%$ greater than that of the Tri-Glide PM with single link movement. Similarly, the Tripod PM has $29.47 \%$ lesser MP tilt than the Tri-Glide PM for the Type II singularity with two links (simultaneous) movement.

- In Type III singularity, the MP tilt about the $\mathrm{x}$ axis of the Tripod PM is $19.84 \%$ greater than that of the Tri-Glide PM. Similarly, the MP tilt about y axis of the Tripod PM is also $48.17 \%$ greater than that of the Tri-Glide PM.

- The pin joint torques and forces were simulated for the two Tripod and Tri-Glide PMs. From the results it is observed that, the Tripod PM has $92.44 \%$ lesser pin joint force for Type I singularity, 91.69\% Type II singularity and $35.56 \%$ Type III singularity than the Tri-Glide PM.

- The Tri-Glide pin joint forces and torque values are found to be higher than those of the Tripod at the singular positions. This may be due to the larger displacement of nut in the singularity II and III. In Type I singularity, the singular position is obtained earlier than the Tripod structure, but the resisting force due to the non actuated links of the Tri-Glide PM is found to be higher.

- Based on the above concluding remarks, the designer can design the PMs based on his/her requirements.

- Further, the dynamic study of the discussed PMs will be carried out for various types of angular bone drilling in medical applications. 


\section{REFERENCES}

1. Fan Zhang, Dan Zhang, Jianguo Yang and Beizhi Li (2005), Inverse kinematics and dynamics of the 3-RRS parallel platform, Proceedings of the IEEE International Conference on Robotics and Automation, Niagra falls, Canada, pp.2117-2122, 2005.

2. Xin-Jun Liu and Jongwon Kim (2002) A new three degree of freedom parallel manipulator. Proceedings of the IEEE international conference on Robotics and Automation 1155-1160.

3. Anjan Kumar Dash, Ming Chen, Song Huat Yeo and Guilin Yang (2003) Singularity - free path planning of parallel manipulators using Clustering Algorithm and Line Geometry. Proc. IEEE, International Conference on Robotics and Automation 761-766.

4. Xin-Jun Liu, Jongwon Kim and Kun-Ku Oh (2003) Singularity analysis of the half parallel manipulator with revolute actuators. Proceedings of the 2003 IEEE International Conference on Robotics and Automation 767-772.

5. Guanfeng Liu, Yunjiang Lou and Zexiang Li (2003) Singularities of parallel manipulators: A geometric treatment. IEEE Transactions on Robotics and Automation 19: 579- 594.

6. Xiang Cheng, Huang, Y.M, Gao, F, Zhang, Y.G and Peng, Z.B (2004) Study on kinematic characteristic and singularities of a 3 - DOF parallel robot. Proceedings of the third International Conference on Machine learning and Cybernetics 5: 2870-2873.

7. Si-Jun Zhu, Zhen Huang and Ming-Yang Zhao (2007) Singularity analysis for a 5-dof fully symmetrical parallel manipulator 5-RRRRR IEEE International Conference on Robotics and Automation 1189-1194.

8. Daniel Kanaan, Philippe Wenger and Damien Chablat (2008) Singularity analysis of limited-dof parallel manipulators using Grassmann-Cayley algebra.11th International Symposium on Advances in Robot Kinematics 1-10.

9. Flavio Firmani and Ron, P. Podhorodeskiv (2009) Singularity analysis of planar parallel manipulators based on forward kinematic solutions. Mechanism and Machine Theory 44: 1386-1399.

10. Ruggiu, M. (2009) Positional analysis, workspace, and optimization of a 3-PPS spatial manipulator. ASME, Journal of Mechanical Design 131:501-510.

11. Clement, M. Gosselin, Louis Perreault and Charles Vaillancourt "Simulation and computer aided design of spherical parallel manipulators", Proceedings on Engineering in harmony with ocean, Vol. 2, pp. 301-306, 1993.

12. Merlet, J.P, "Optimal design of robots", Online Proceedings of Robotics: Science and Systems. Boston, pp. 1-8, 2005.

13. Sameer Joshi and Lung Wen Tsai "A comparison study of two 3 - DOF parallel manipulators: one with three and the other with four supporting legs", IEEE transactions on Robotics and Automation, Vol.19, pp. 200-209, 2003.

14. Arockia Selvakumar, A, Karthik, Naresh Kumar, Sivaramakrishnan R (2012) "Kinematic and Singularity analysis of 3PRR Manipulator", Advanced Material Research, 403, 5015-5021.

15. Arockia Selvakumar, A, Praveen Babu, Sivaramakrishnan R (2012) "Simulation and Singularity analysis of 3PRS Manipulator." IEEE International Conference on Mechatronics and Automation, 2203-2207.

16. Arockia Selvakumar, A, Harish, Saket (2018) "Experimental investigation on position analysis of 3-RPR parallel manipulators." Journal of Advanced Research in dynamical systems, 10 (5), 556-564.

17. A Arockia Selvakumar, G Hallale, LP Kalyan (2018), Kinematic Analysis of a T-3R Manipulator for Material Handling Applications, Journal of Advanced Research in dynamical systems, 10 (3), 40-48.

\section{AUTHORS PROFILE}

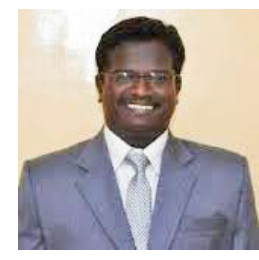

Dr. Arockia Selvakumar A, is a member of Design and Automation Research group at the Vellore Institute of Technology, Chennai where he is working as Senior Associate Professor of School of Mechanical and Building Sciences. He has an exceptional publication record with over $100+$ citations (100 archival journal papers, 45 papers in major conferences, 5 book chapters and 9 patents granted and applied). He has supervised to date many graduate students, 100+ UG, 50+ PG and 5 Ph.D.(guiding) Students. He holds a lifetime membership of RSI and a member of IEEE. His field of interest includes Robotics and Automation, IOT, Bio-Mechanics and Finite Element analysis. 